\title{
What really happens to tuberculosis patients classified as lost to follow-up in West Yorkshire?
}

M Day (matthew.day2@nhs.net) ${ }^{1,2}$, A Middlemiss' ${ }^{1}$, J Thorpe ${ }^{1}$, E Okereke ${ }^{1}$

1. Health Protection Agency, Yorkshire and the Humber, Leeds, United Kingdom

2. School of Health and Related Research, University of Sheffield, Sheffield, United Kingdom

Day M, Middlemiss A, Thorpe J, Okereke E. What really happens to tuberculosis patients classified as lost to follow-up in West Yorkshire?. Euro Surveill. 2012;17(38): pii=20278. Available online: http://www.eurosurveillance.org/ViewArticle.aspx?Articleld=20278

Tuberculosis (TB) patients who do not complete treatment pose a potential public health risk. In West Yorkshire, local clinicians suspected that this risk was overestimated by the national Enhanced Tuberculosis Surveillance system. We audited patients who failed to complete treatment and were categorised as lostto-follow-up (LTFU) between 2004 and 2008, using a combination of hand searching existing records and obtaining additional information from clinicians. In the study period 2,031 TB cases with reported outcome were notified in West Yorkshire, $23 \%(n=474)$ did not complete treatment, and 199 (42\%) of those were categorised as LTFU 12 months after notification. Of these $199,49 \%(n=98)$ remained LTFU after the audit, $51 \%(n=101)$ were re-classified to the following categories: $24 \%(n=47)$ transferred abroad, $16 \%(n=31)$ recommenced and completed treatment, $6 \%(n=13)$ transferred to another clinic in the United Kingdom (UK), and $5 \%(n=10)$ died. These patients therefore no-longer posed a public health risk. Further training for clinicians to improve accuracy of outcome reporting has been initiated. Nationally, the collection of treatment outcome data needs to be strengthened and extending the follow-up for treatment outcome monitoring should be considered.

\section{Background}

The most important intervention for the control of tuberculosis (TB) is effective treatment of infectious cases [1]. Failure to complete treatment poses a significant public health risk through disease reactivation, increased transmission, and development of drug-resistance.

Treatment success measured by a standardised process of treatment outcome monitoring is one of the pillars of TB control. The World Health Assembly (WHA) passed a resolution in 1991, adopting the target to cure at least $85 \%$ of TB cases as one of two global targets [2]. In the European Union (EU), TB case notification rates are among the lowest in the world, declining by $15.2 \%$ between 2005 and 2010 [3]. However, there was a concurrent decline in treatment completion rates in the region, declining from $72.5 \%$ in 2005 to $68.7 \%$ in
2010 among new diagnosed cases, the lowest TB treatment success rate in the world and short of the WHA resolution target [3]. Eleven per cent of newly diagnosed laboratory-confirmed pulmonary cases are lost to follow-up (LTFU) [3]. There has been limited research into this phenomenon in the region and investigation is required to determine the underlying factors and implementing measures required to address it.

In England, the Chief Medical Officer adopted the WHA target in their TB Action Plan 2004 [4], and most recently, TB treatment completion has been included as a key indicator in the Public Health Outcome Framework for England [5].

The Enhanced Tuberculosis Surveillance System (ETS) collates detailed epidemiological, clinical and microbiological information on TB cases in England, Wales and Northern Ireland, including treatment outcomes. In line with World Health Organization (WHO) recommendations [6], treatment outcome is recorded 12 months after notification, and then again at 24 months for those who are still on treatment at 12 months. The Health Protection Agency publishes these results annually and measures performance against the national target of $85 \%$ treatment completion at 12 months from notification [4]. In 2010, the national treatment completion rate was $78 \%[7]$.

A UK study argued that the current categories used to monitor treatment outcome (Figure 1) are limited in capturing patients' true outcomes, as it is not collected properly in all cases [8]. Refining the categories used in the current surveillance system to focus on patients' final true outcomes could significantly improve the measured treatment completion rates [8]. This in turn would provide a more accurate picture of those patients who pose a genuine risk to public health.

In West Yorkshire, the treatment completion rate in 2008 was $75 \%$ [9], and $78 \%$ in 2009 and $2010[10,11]$ well below the national average and target. The $25 \%$ not completing treatment include patients classified as LTFU, defined as the failure to obtain contact with 


\section{TUBERCULOSIS TREATMENT OUTCOME SURVEILLANCE FORM ENGLAND, WALES \& NORTHERN IRELAND}

This form should be completed 12 months after start of treatment, but can be filled in earlier for patients that have completed treatment, died, were misdiagnosed (not TB) or had their treatment stopped (and not restarted). For patients still on treatment at 12 months the form should also be completed at 24 months. For more information see the guidance notes attached / on reverse.

\section{Section A. PATIENT INFORMATION}

\begin{tabular}{|c|c|c|c|}
\hline ID: & Surname: & Forename: & \\
\hline $\begin{array}{l}\text { Local ID: } \\
\text { Street: }\end{array}$ & Date of birth: & $\begin{array}{l}\text { Age (years): } \\
\text { Town: }\end{array}$ & Sex: \\
\hline Post code: & PCT/Health board (residence): PCT & & \\
\hline $\begin{array}{l}\text { Date of case report: } \\
\text { Hospital: }\end{array}$ & Date treatment started: & $\begin{array}{l}\text { Patient's Consultant: } \\
\text { Case manager: }\end{array}$ & \\
\hline
\end{tabular}

Section B. TRANSFER DETAILS

If the patient's care was transferred to another clinical team please give details, then - Go to section D

Clinician:.

Hospital/Trust:.

\section{Section C. TREATMENT OUTCOME}

Name and post of person completing this form:

Date of completion of this form:

Did the patient complete a full course of therapy within 12/24 months of starting treatment?

1. $\square$ Yes If Yes, date of end of treatment:........................

treatment regimen used: $\square$ standard (2 months HRZE and 4 of HR)

$\square$ other (please specify)....

2. $\square$ No - Patient did not complete treatment within $12 / 24$ months because (please tick appropriate boxes):

$\square$ Treatment stopped: $\quad \square$ Patient found not to have TB* (including atypical mycobacterial infection) or

$\square$ Other reason

Or $\square$ Patient died before or while on treatment (including post mortem diagnosis).

If yes, date of death: $\quad \ldots \ldots . . . \ldots / \ldots \ldots . . . . \quad$ Please indicate whether:

$\square$ TB caused death or

$\square$ TB contributed to death or

$\square$ TB incidental to death or

Relationship between TB and death unknown

Or $\square$ Planned course of treatment exceeds 12 months as a result of: $\square$ initial drug resistance

Or $\square$ Planned course of treatment was interrupted as a result of: $\square$ intolerance / side effects

$\square$ other

Or $\square$ Planned course of treatment changed as a result of:

$\square$ intolerance / side effects
$\square$ Initial drug resistance
$\square$ development of new drug resistance
$\square$ failure to culture convert
$\square$ poor clinical response to treatment
$\square$ other

3. $\square$ Patient was lost to follow-up before the end of treatment because: $\square$ patient left the UK

4. $\square$ Unknown - only tick if treatment details are unavailable (e.g. lost patient notes)

* This case should be de-notified from the electronic surveillance system 
Tuberculosis patient treatment outcomes before and after audit, West Yorkshire, September 2010-March 2011 (n=2,076)

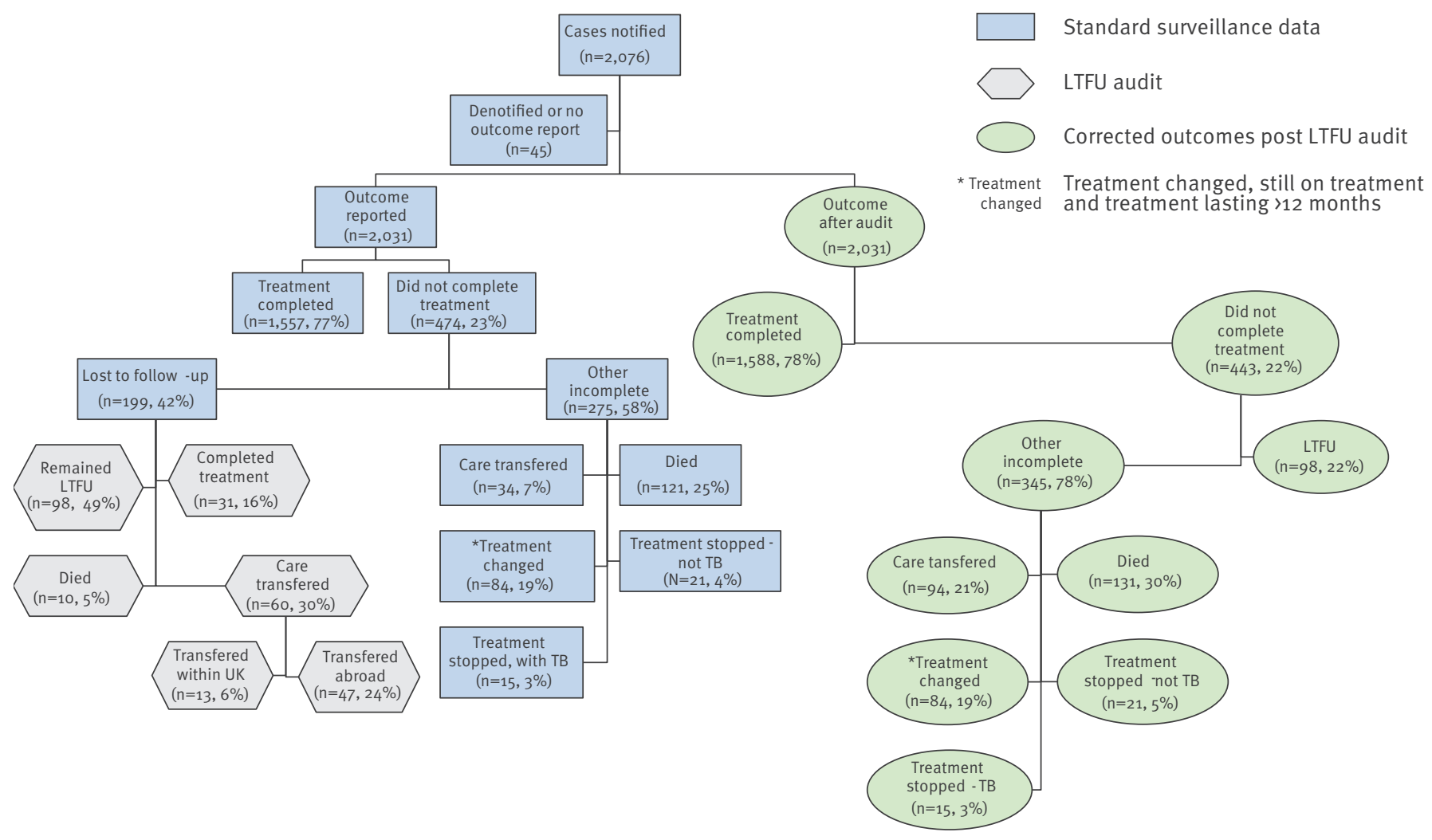

LTFU: lost to follow-up; TB: tuberculosis, UK: United Kingdom.

the patient before the end of treatment [12], so that the treatment outcome is not known. Patients included in this category are considered to be a potential public health risk due to their unknown outcome, whereabouts or disease status. Alongside the published evidence on the categories used to classify treatment outcomes [8], the West Yorkshire TB Network were concerned that the LTFU category was being overused in the absence of more accurate descriptors and that in reality some of the patients who were classified as LTFU had completed treatment. This meant that the surveillance system reported treatment completion rates that were lower than clinicians would expect, and from a public health perspective the true risk to public health posed by LTFU patients was being overestimated based on current surveillance data. Our audit therefore focussed on addressing this issue to understand what really happens to TB patients classified as being LTFU.

\section{Methods}

The audit was conducted over a period of seven months from September 2010 to March 2011. Records of all TB cases notified in West Yorkshire between 2004 and 2008 were extracted from the ETS and treatment outcomes reviewed. Records for those classified as LTFU were extracted. Demographic and risk factor information was compared to patients classified as having completed treatment. Paper records for patients classified as LTFU, were hand searched. Any additional information contained in the free text section of the reporting form that had not previously been transferred to the ETS was gathered. Where no additional information was recorded on the outcome form, TB clinical teams who had been responsible for the treatment of the case were contacted to clarify the true outcome. The collated information was analysed using standard treatment outcome categories (Figure 1) and further relevant categories that were developed during the analysis based on the collected information, such as recommenced and completed treatment.

\section{Results}

Between 2004 and 2008 2,031 TB cases were notified in West Yorkshire (Figure 2). Of those, 474 (23\%) were reported as not having completed treatment. Twelve months after notification, 199 (42\%) of those not completing treatment were categorised as LTFU.

LTFU patients were more often male and older than those who completed treatment, although these 
Baseline characteristics comparing patients classified as lost to follow-up to those who had completed treatment

\begin{tabular}{|c|c|c|}
\hline & LTFU $n=199$ & Complete $n=1,557$ \\
\hline Male n (\%) & $110(55)$ & $795(51)$ \\
\hline Female n (\%) & $89(45)$ & $758(49)$ \\
\hline Unknown sex n (\%) & $o(0)$ & $4(0.3)$ \\
\hline Median age in years ( $95 \%$ confidence interval) & $35(32-39)$ & $33(32-34)$ \\
\hline$\%$ pulmonary TB ( $95 \%$ confidence interval) & $48(40-55)$ & $48(46-51)$ \\
\hline \multicolumn{3}{|l|}{ Most common ethnic groups $\mathrm{n}(\%)$} \\
\hline Pakistani & $78(39)$ & $703(43)$ \\
\hline Black African & $36(18)$ & $228(15)$ \\
\hline Indian & $30(15)$ & $200(13)$ \\
\hline White & $26(13)$ & $228(15)$ \\
\hline
\end{tabular}

LTFU: lost to follow-up; TB: tuberculosis.

differences, along with ethnicity and site of TB were not statistically significant (Table).

The hexagonal boxes in Figure 2 show that of the 199 patients initially classified as LTFU, 98 (49\%) remained LTFU after the audit. No further information was available relating to these patients' outcomes. Of the remaining 101 patients, 47 (24\%) had transferred abroad, 31 $(16 \%)$ had recommenced and completed treatment, 10 (5\%) had died and $13(6 \%)$ transferred to another UK clinic.

India and Pakistan were the countries most frequently reported as the destination for those transferring abroad (20/47), which is in keeping with the cultural background of TB cases in West Yorkshire, where over a third of reported cases have a Pakistani ethnic origin [9]. However, a wide range of destinations across Asia and Africa were also noted. Of the 47 patients who left the UK, only $10(21 \%)$ were reported to have left with a full supply of treatment.

The circular boxes in Figure 2 show that after excluding cases inaccurately categorised, who recommenced and subsequently completed treatment, and those who transferred abroad, the proportion of all TB cases in West Yorkshire between 2004 and 2008 that were truly LTFU was not $10 \%(n=199 / 2,031)$ but $5 \%(n=98 / 2,031)$ of this five-year cohort.

\section{Discussion}

In West Yorkshire, between 2004 and 2008, more patients successfully completed treatment than existing surveillance data indicated, which implies that the risk to public health from treatment failure may have been over-estimated.

Some of this variation in reporting is due to the fact that reporting clinicians did not always select the appropriate option in the reporting form. This could have occurred for two reasons: the first is through classification error where clinicians had access to the correct information regarding the patients' outcome but completed the wrong section on the Treatment Outcome Monitoring (TOM) form; the second is that the clinician simply did not have the relevant information to hand at the time of completing the form, which resulted in misclassification. This second scenario is particularly relevant to the many patients reported as LTFU at 12 months who were subsequently found, recommenced and completed treatment or transferred to services abroad.

There is currently little literature examining the validity of the LTFU category in relation to national surveillance systems. A recent study by Ditah et al. [8] focused on the categories used for TB surveillance and consequently cannot be generalised to allow comparison with our work. Ditah et al's study analysed predominantly patients whose treatment was interrupted or lasted longer than a year. It did not explore what happened to patients who were LTFU, simply categorising these patients as 'treatment failures'. As a result, the study does not consider the public health risk posed by this group of patients.

There is a wider range of literature relating to the risk factors for failing to complete treatment for TB. These include social deprivation, illiteracy, history of incarceration, older age, white ethnicity, and a history of substance misuse [13-15]. Our audit was unable to consider these wider risk factors in depth due to incomplete data. The data we analysed predates the collection of this information in the ETS system. Further work looking at the distribution of these risk factors amongst TB cases LTFU should be a priority. Our results showed no significant differences in terms of sex, age, ethnicity, or site of TB between those who completed treatment and those who were classified as LTFU.

Our audit suggests that there is a need to consider refining treatment outcome reporting to more 
accurately reflect risk to public health. Our results showed that $16 \%$ of patients initially classified as LTFU subsequently recommenced and completed treatment, often many months after the 12 month deadline. Whilst the current treatment outcome monitoring form (Figure 1) states clearly that for patients still on treatment at 12 months, the form should be also be completed at 24 months, this does not capture patients who had stopped treatment prior to 12 months, were classified as LTFU and then recommenced treatment after the 12-month period. Our results show similarities to a study conducted in London [16], which re-reviewed treatment outcomes at 24 months after treatment initiation. That study showed that the group of patients classified as treatment failure at two years constituted mainly patients transferring abroad, transferring to another clinic, and those that died. Our audit highlights that new information often becomes available for patients classified as LTFU after the 12-month deadline. Consideration should be given to extending the deadline for treatment outcome reporting or changing the 24-month follow-up to include not just patients continuing treatment for longer than 12 months, but LTFU patients as well.

Our audit also suggests that significant numbers of our patients had interrupted treatment, resulting in significantly extended treatment regimes. Treatment interruption contributes to increased drug resistance in TB patients [17]. This suggests that more effort should be made at the time of diagnosis to identify patients at risk of treatment interruption and to use enhanced case management protocols and incentives to mitigate this risk. This will require increased investment in TB clinical teams, specifically community-based TB nurses and case mangers.

Locally, an outcome of this audit has included further training for clinicians to improve accuracy of outcome reporting. Nationally and internationally, a sustained focus on, and awareness of, the importance of enhanced TB surveillance is essential in reducing the public health threat posed by LTFU cases.

Risk factors for poor treatment adherence were not routinely collected by ETS during the period audited, and as such only unvalidated information from TB nursing records was available. This information suggested that poor treatment adherence was predicted for some of these patients and that, had resources been available, many of these patients would have been put on enhanced case management regimes at the time of diagnosis, such as directly observed therapy. The national ETS system introduced the collection of information about social risk factors for all notified TB cases in 2009, and therefore completeness levels are low but improving [8]. Analysing this improving information and conducting further work to quantify the risk of a patient becoming LTFU is important and should be a priority for the Health Protection Agency and in the future, Public Health England.
This audit only considers outcomes for patients notified in West Yorkshire (population 2.2 million). It is therefore important to understand if treatment outcome reporting in West Yorkshire differs significantly from the national pattern. A total of 379 patients $(4.6 \%$ of reported cases) were classified nationally as LTFU in 2009 [7]. This rate is closer to the corrected figure obtained after our audit, suggesting that the overestimation of LTFU may be a local concern. At European level, results from the England, Wales and Northern Ireland TOM are collated by the European Surveillance System (TESSy). A recent report raised concern regarding the variations in TOM definitions and data collection methods between EU Member States, which impact upon results reported by TESSy [18]. Our results therefore emphasise that further work is necessary to understand possible national variations and should also ensure that TOM outcomes reported from national surveillance systems are consistent between countries and, most importantly, reflect the true outcomes of patients. 


\section{References}

1. Abubakar I, Lipman M, Anderson C, Davies P, Zumla A. Tuberculosis in the UK - Time to regain control. BMJ. 2011;343:d4281.

2. Stop TB Partnership and World Health Organization (WHO). The global plan to stop TB 2006-2015. Actions for life: towards a world free of tuberculosis. Geneva: WHO; 2006. WHO/HTM/ STB/2006.35. Available from: http://www.stoptb.org/assets/ documents/global/plan/GlobalPlanFinal.pdf

3. European Centre for Disease Prevention and Control (ECDC)/ World Health Organization Regional Office for Europe. Tuberculosis surveillance and monitoring in Europe 2012. Stockholm: ECDC; 2012. Available from: http://ecdc.europa.eu/ en/publications/Publications/1203-Annual-TB-Report.pdf

4. Stopping Tuberculosis in England. An Action Plan from the Chief Medical Officer. London: Department of Health; 2004. Available from: http://www.dh.gov. uk/en/Publicationsandstatistics/Publications/ PublicationsPolicyAndGuidance/DH_4090417

5. Healthy lives, healthy people: Improving outcomes and supporting transparency. London: Department of Health; 2012. Available from: http://www.dh.gov. uk/en/Publicationsandstatistics/Publications/ PublicationsPolicyAndGuidance/DH_132358

6. Veen J, Raviglione M, Rieder HL, Migliori GB, Graf P, Grzemska $M$, et al. Standardised tuberculosis treatment outcome monitoring in Europe. Recommendations of a Working Group of the World Health Organisation (WHO) and the European Region of the International Union against Tuberculosis and Lung Disease (IUATLD) for uniform reporting by cohort analysis of treatment outcome in tuberculosis patients. Eur Respir J. 1998;12(2):505-10.

7. Tuberculosis in the UK: Annual report on tuberculosis surveillance in the UK 2010. London: Health Protection Agency Centre for Infections; 2010. Available from: http:// www.hpa.org.uk/web/HPAweb\&HPAwebStandard/ HPAweb_C/1287143581697

8. Ditah IC, Reacher M, Palmer C, Watson IM, Innes I, Kruijshaar $\mathrm{ME}$, et al. Monitoring tuberculosis treatment outcome: analysis of national surveillance data from a clinical perspective. Thorax. 2008;63(5):440-6.

9. Okereke E, Thorpe J. Tuberculosis in Yorkshire and the Humber region 2009. A report from the HPA in Yorkshire and The Humber. London: Health Protection Agency; 2010. Available from: http://www.hpa.org.uk/webc/HPAwebFile/ HPAweb_C/1296682353074

10. Okereke E, Thorpe J. Tuberculosis in Yorkshire and the Humber region 2010: A report from the HPA in Yorkshire and The Humber. London: Health Protection Agency; 2011.

11. Health Protection Agency (HPA). New Figures Show Rise in TB Cases. TB Global Emergency 'A Reality in Yorkshire and the Humber'. London: HPA; September 2012. Available from: http://www.hpa.org.uk/AboutTheHPA/ WhatTheHealthProtectionAgencyDoes/LocalServices/ YorkshireHumber/YorkshireAndHumberPressReleases/ Yorks120323NewFiguresShowRiseinTBCases

12. Health Protection Agency (HPA). Web based Enhanced Tuberculosis Surveillance (ETS) Data Dictionary. Version 2. London: HPA; September 2012.

13. Story A, Murad S, Verheyen M, Roberts W, Hayward AC. Tuberculosis in London-the importance of homelessness, problem drug use and prison. Thorax. 2007;62(8):667-71.

14. Anderson C, Story A, Brown T, Drobniewski F, Abubakar I. Tuberculosis in UK prisoners: a challenge for control. Epidemiol Community Health. 2010;64(4):373-6.

15. Story A, van Hest $R$, Hayward A. Tuberculosis and social exclusion. BMJ. 2006;333(7558):57-8.

16. Conaty SJ, Dart S, Hayward AC, Lipman MC. Reasons for low reported treatment success in notified tuberculosis patients from a London hospital according to new outcome reporting. Commun Dis Public Health. 2004;7(1):73-6.

17. World Health Organization (WHO). Multidrug and Extensively Drug-resistant TB (M/XDRTB): 2010 global report on surveillance and response. Geneva: WHO; 2010. WHO/ HTM/TB/2010.3. Available from: http://whqlibdoc.who.int/ publications/2010/9789241599191_eng.pdf

18. Manissero D, Hollo V, Huitric E, Kodmon C, Amato-Gauci A. Analysis of tuberculosis treatment outcomes in the European Union and European Economic Area: efforts needed towards optimal case management and control. Euro Surveill. 2010;15(11):pii=19514.Available from: http://www. eurosurveillance.org/ ViewArticle.aspx?Articleld=19514 